\title{
Mechanism for the endocytosis of spherical nucleic acid nanoparticle conjugates
}

\author{
Chung Hang J. Choi ${ }^{\mathrm{a}, \mathrm{b}, 1}$, Liangliang Hao ${ }^{\mathrm{a}, \mathrm{b}, \mathrm{c}, 1}$, Suguna P. Narayan ${ }^{\mathrm{a}, \mathrm{d}}$, Evelyn Auyeung ${ }^{\mathrm{a}, \mathrm{e}}$, and Chad A. Mirkin ${ }^{\mathrm{a}, \mathrm{b}, \mathrm{d}, \mathrm{e}, \mathrm{2}}$ \\ ${ }^{a}$ International Institute for Nanotechnology, Departments of ${ }^{b}$ Chemistry, ${ }^{d}$ Biomedical Engineering, and ${ }^{e}$ Materials Science and Engineering, \\ and 'Interdepartmental Biological Sciences Program, Northwestern University, Evanston, IL 60208
}

Contributed by Chad A. Mirkin, March 26, 2013 (sent for review February 20, 2013)

Intracellular delivery of nucleic acids as gene regulation agents typically requires the use of cationic carriers or viral vectors, yet issues related to cellular toxicity or immune responses hamper their attractiveness as therapeutic candidates. The discovery that spherical nucleic acids (SNAs), polyanionic structures comprised of densely packed, highly oriented oligonucleotides covalently attached to the surface of nanoparticles, can effectively enter more than $\mathbf{5 0}$ different cell types presents a potential strategy for overcoming the limitations of conventional transfection agents. Unfortunately, little is known about the mechanism of endocytosis of SNAs, including the pathway of entry and specific proteins involved. Here, we demonstrate that the rapid cellular uptake kinetics and intracellular transport of SNAs stem from the arrangement of oligonucleotides into a 3D architecture, which supports their targeting of class $A$ scavenger receptors and endocytosis via a lipid-raft-dependent, caveolae-mediated pathway. These results reinforce the notion that SNAs can serve as therapeutic payloads and targeting structures to engage biological pathways not readily accessible with linear oligonucleotides.

S pherical nucleic acids (SNAs) represent an emerging class of nucleic acids distinct from linear nucleic acids in the context of gene regulation $(1,2)$. These nanostructures, although polyanionic like their linear counterparts, can naturally traverse the negatively charged cell membrane and enter cells. By contrast, linear nucleic acids of the same sequence typically require the use of cationic transfection agents to overcome the coulombic repulsive forces between the cell membrane and their phosphate backbone. For example, when C166 (mouse endothelial) cells are treated with Cyanine 5 (Cy5)-labeled, single-stranded oligonucleotides $(1 \mu \mathrm{M}$, Cy5-ssDNAs; sequence information listed in Table S1), confocal microscopy after $2 \mathrm{~h}$ of incubation revealed no appreciable uptake (Fig. 1A). However, when the cells are treated with Cy5-SNAs made from Cy5-ssDNAs of identical sequence covalently attached to the surface of spherical gold nanoparticles (AuNPs) (10 nM particles with 100 oligonucleotides per particle; Fig. S1), significant intracellular accumulation could be observed (Fig. 1B). Note that the SNAs are at 1/100 the concentration of the free oligonucleotides, and on a total DNA basis, both experiments contain identical nucleic acid amounts. Even by complexing oligonucleotides of the same sequence with DharmaFECT, a conventional cationic transfection agent, the resultant polycation/ oligonucleotide complexes are primarily localized on the cell membrane without significant intracellular accumulation over the same $2 \mathrm{~h}$ time period (Fig. $1 C$ ). Thus, SNAs appear to mediate the endocytosis of oligonucleotides using a mechanism that is different from the electrostatic interactions exploited by many cationic transfection agents. We further monitored the endocytosis of Cy5-labeled "hollow SNAs," 3D oligonucleotide-based constructs obtained by crosslinking multiple alkyne-containing Cy5-ssDNAs on the surface of an AuNP core that was subsequently dissolved with $\mathrm{KCN}$ (3). Hollow SNAs enter cells at a rate and to an extent similar to those observed for Cy5-SNAs with the core, reinforcing the notion that the 3D oligonucleotide shell, and not the nature of the NP core, is the dominant factor governing the endocytosis of SNAs (Fig. 1D).

Since SNAs have been heavily used in intracellular detection (4) and gene regulation (5) applications, it is imperative that the biological mechanism that governs their endocytosis be determined. In this work, we have monitored the cellular uptake kinetics and intracellular transport of SNAs, determined their primary entry pathway, and identified the primary receptor subclass that mediates their internalization into mammalian cells.

\section{Results and Discussion}

Cellular Uptake Kinetics and Intracellular Transport. To probe the biological effects due to the 3D arrangement of oligonucleotides that comprise SNAs, we first characterized their cellular uptake kinetics and intracellular transport. We performed all cell culture experiments by incubating C166 cells with SNAs formulated in Opti-MEM I reduced serum media (OptiMEM) to exclude the effects of serum proteins on the endocytosis mechanism. After incubation of cells with SNAs over different durations of time, we used inductively coupled plasma mass spectrometry (ICP-MS) to quantify the rate of SNA cellular association. The most rapid SNA association occurs within the first $30 \mathrm{~min}$, as evidenced by the rapid rise in gold content (Fig. $2 A$ ). After 5 min of incubation, $\sim 3.6 \times 10^{4}$ NPs are associated with each cell. Over the next $30 \mathrm{~min}$, rapid accumulation of particles continues, albeit at a slower rate. After $4 \mathrm{~h}$ of incubation, there are $\sim 2.7 \times 10^{5}$ SNAs associated with each cell. Note that the cell pellets used to generate the ICP-MS data are red, due to the plasmon resonance of the AuNPs at $520 \mathrm{~nm}$. This red color increases steadily over the first $4 \mathrm{~h}$ of incubation (Fig. $2 B$ ). Next, we used confocal microscopy to visualize the transport of Cy5-labeled SNAs (Cy5-SNAs) across the cell membrane and into the cytoplasm (Fig. 2C). At the initial stages (15 min after introduction of SNAs to cells), the fluorescence signal rises at the cell periphery. Over the next hour, the fluorescence signal moves inward, indicating particle internalization. To characterize the subcellular localization of SNAs, we further used transmission electron microscopy (TEM) to image C166 cells incubated with SNAs (Fig. 2D). After incubation for $15 \mathrm{~min}$, individual SNA particles are found inside invaginations or small vesicles $(\sim 50 \mathrm{~nm}$ in diameter) in the cell membrane, indicating binding and entry of SNAs into cells. Intracellular SNA accumulation becomes more pronounced starting from $30 \mathrm{~min}$ after incubation. After $1 \mathrm{~h}$, SNAs are found in larger vesicles $(100-250 \mathrm{~nm}$ in diameter) that are reminiscent of early endosomes. Indeed, confocal immunofluorescence reveals colocalization between the fluorescent signals of Cy5-SNAs and early endosome antigen-1 (EEA1) at the same time point (Fig. $2 E$ ), confirming sorting of SNAs to early endosomes. Based on both confocal and TEM imaging data, particles never enter the nucleus over the entire incubation period. Thus, the ICP-MS, confocal imaging, and TEM data collectively illustrate three key events that define the endocytosis of SNAs: $(i)$ binding to the cell membrane, (ii) uptake via invaginations, and (iii) sorting into early endosomes.

\footnotetext{
Author contributions: C.H.J.C., L.H., and C.A.M. designed research; C.H.J.C., L.H., S.P.N., and E.A. performed research; C.H.J.C., L.H., S.P.N., E.A., and C.A.M. analyzed data; and C.H.J.C., L.H., and C.A.M. wrote the paper.

The authors declare no conflict of interest.

${ }^{1}$ C.H.J.C. and L.H. contributed equally to this work.

${ }^{2}$ To whom correspondence should be addressed. E-mail: chadnano@northwestern.edu.
}

This article contains supporting information online at www.pnas.org/lookup/suppl/doi:10. 1073/pnas.1305804110/-/DCSupplemental. 


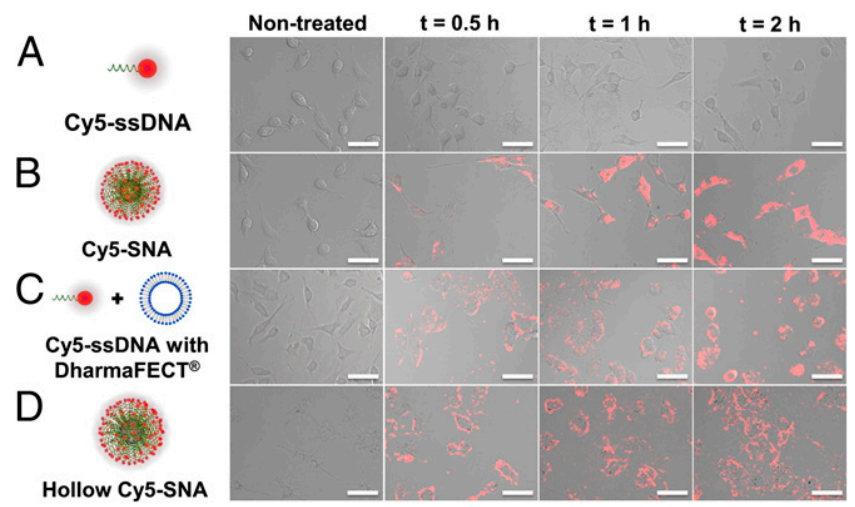

Fig. 1. Effect of oligonucleotide dimensionality on endocytosis. (A) Cy5ssDNAs cannot enter C166 cells in amounts detectable by confocal microscopy. $(B)$ With their 3D architecture, Cy5-SNAs that contain the same total DNA concentration can readily enter cells, starting from binding to the cell membrane $30 \mathrm{~min}$ after incubation to populating the cytosol $2 \mathrm{~h}$ after incubation. (C) Encapsulation of Cy5-ssDNAs with DharmaFECT led to the tracing of the cell border by Cy5-ssDNAs without significant intracellular accumulation. $(D)$ Hollow Cy5-SNAs enter cells with similar kinetics and extent as Cy5-SNAs.

Caveolae/Lipid-Raft as the Major Pathway of Endocytosis. It is important to determine the pathway of endocytosis that is consistent with the rapid uptake kinetics and intracellular transport of SNAs. Since SNAs are localized in vesicles that contain EEA1, such as early endosomes, we focused our attention on clathrin-mediated endocytosis and lipid-raft-mediated endocytosis, both of which can shuttle endocytosed biological molecules to early endosomes (6). We first examined whether SNA endocytosis is clathrin-mediated. By confocal immunofluorescence, SNAs weakly colocalize with clathrin heavy chain, the basic subunit of the clathrin coat (Fig. $\mathrm{S} 2 A$ ). Based on ICP-MS measurements made in parallel with the microscopy studies but using different cell samples, pretreatment of C166 cells with chlorpromazine, a pharmacological inhibitor that disrupts the formation of clathrin-coated pits (7), merely reduced the uptake of SNAs by $12 \%$ (Fig. S2A). To probe the role of clathrin with higher specificity, we prepared stably transfected clathrin-deficient C166 cells (denoted "CLTC-DF") by using lentiviruses to deliver GIPZ lentiviral vectors (pGIPZ) that contain a short hairpin RNA (shRNA) sequence for silencing the expression of the CLTC gene, which encodes for clathrin heavy chain (Fig. S4). Compared with control C166 cells that were lentivirally transfected with pGIPZ plasmids that do not contain any shRNA (denoted "pGIPZ-control"), CLTC-DF cells exhibited less than $10 \%$ reduction in endocytosis of SNAs (Fig. 3E). These data led us to exclude clathrin-mediated endocytosis as a major pathway for C166 cells.

Finally, we examined whether SNA endocytosis could be mediated by lipid-rafts. Based on confocal immunofluorescence data, Cy5-SNAs exhibit some colocalization with cholera toxin subunit B (CTX-B), caveolin-1 (CAV-1), and flotillin-1 (FLOT-1) (Fig. $3 A$ ), all resident proteins of lipid-rafts (8). Pretreatment of C166 cells with pharmacological inhibitors that act according to various blocking mechanisms of cholesterol, including methyl- $\beta$ cyclodextrin (which depletes and removes cholesterol) and filipin (which sequesters cholesterol) (9), decreased intracellular accumulation of Cy5-SNAs (Fig. S2B). Remarkably, for filipin-treated cells, Cy5-SNAs are only localized along the cell membrane (as evidenced by the rings of fluorescence) and rarely enter the cytosol (Fig. 3B). Based on ICP-MS data from independent cell samples, pretreatment with methyl- $\beta$-cyclodextrin and filipin reduced the cellular association of SNAs by $49 \%$ and $70 \%$, respectively (Fig. $\mathrm{S} 2 B$ ). We also used TEM to monitor the interaction between SNAs and the cell membrane at the initial stage of endocytosis. After 5 min of incubation, the portion of the cell membrane that contains large amounts of SNAs reveals a darker intensity than portions without SNAs (Fig. 3C), possibly indicating the presence of lipid-raft microdomains that contain high concentrations of lipidated proteins or transmembrane proteins (10). These data suggest that the pathway of endocytosis of SNAs is mediated by lipid-rafts and motivated us to further investigate the specific component of lipid-raft involved in the process. The apparent sensitivity of uptake to pretreatment of $\mathrm{C} 166$ cells by filipin implies the involvement of caveolae (11), characterized by flask-shaped 60-80 nm diameter pits that are abundantly found in the membrane of specific cell types, including endothelial cells (12). Two common classes of endogenous cargo implicated for caveolae-

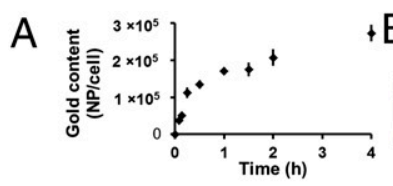

$+\mathrm{B}$
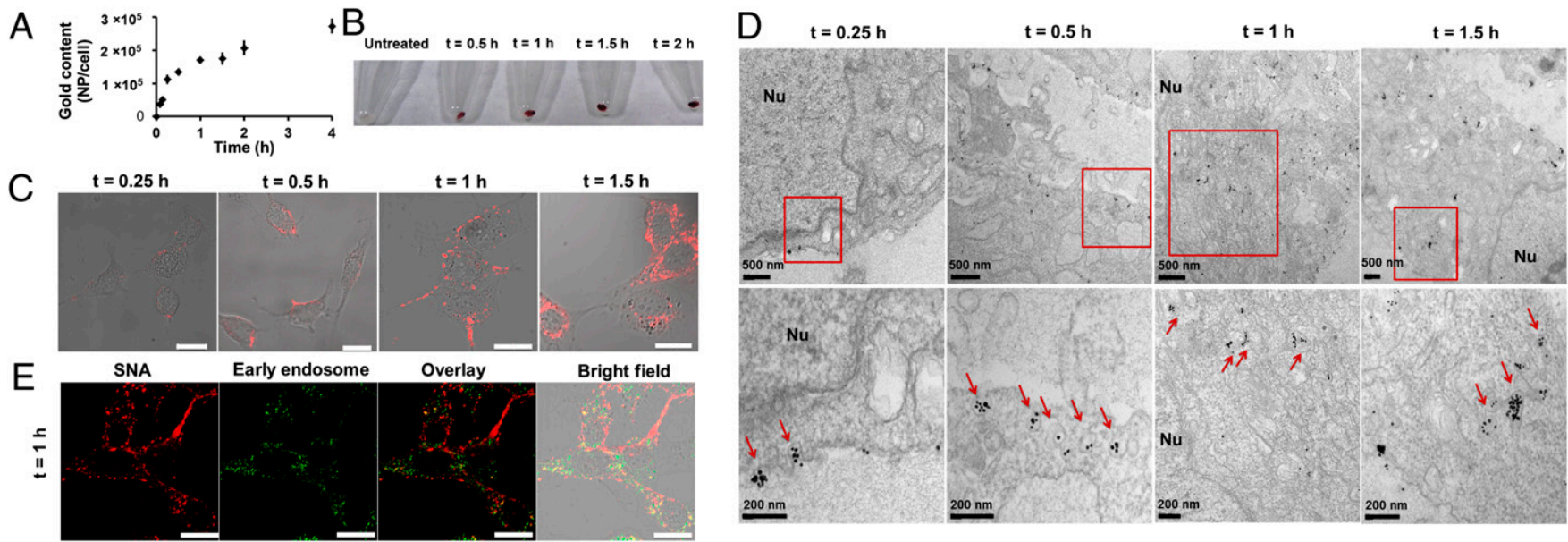

Fig. 2. Cellular uptake kinetics and intracellular transport. $(A)$ ICP-MS measurements of the gold content of C166 cells incubated with SNAs for varying durations of time reveal two distinct phases. The initial portion of the uptake curve (first 30 min) represents SNA binding on the cell membrane. The later portion (after $30 \mathrm{~min}$ ) indicates SNA internalization. (B) Cell pellets reveal a trend of increasing SNA content (red color from plasmon resonance of AuNPs) as a function of incubation time. (C) Time-course confocal images reveal the intracellular transport of SNAs. Cy5-SNAs bind to the cell surface after 15 min of incubation. The fluorescence signals continue to increase during the first $2 \mathrm{~h}$ of incubation. (Scale bar, $20 \mu \mathrm{m}$.) (D) Time-course TEM images reveal the subcellular localization of SNAs. Individual SNAs bind to cell membrane and enter invaginations (arrows) after 15 min of incubation. They enter the cytosol and remain in larger vesicles that resemble early endosomes (100-300 nm; arrows) over the next hour. The second row features enlarged images of the boxed regions of the first. Nu, nucleus. $(E)$ By confocal immunofluorescence, some portions of Cy5-labeled SNAs (red) colocalize with EEA1 (green) with a Manders overlap coefficient of 0.61 (yellow), indicating sorting of SNAs to early endosomes after their entry to cells. (Scale bar, $20 \mu \mathrm{m}$.) 

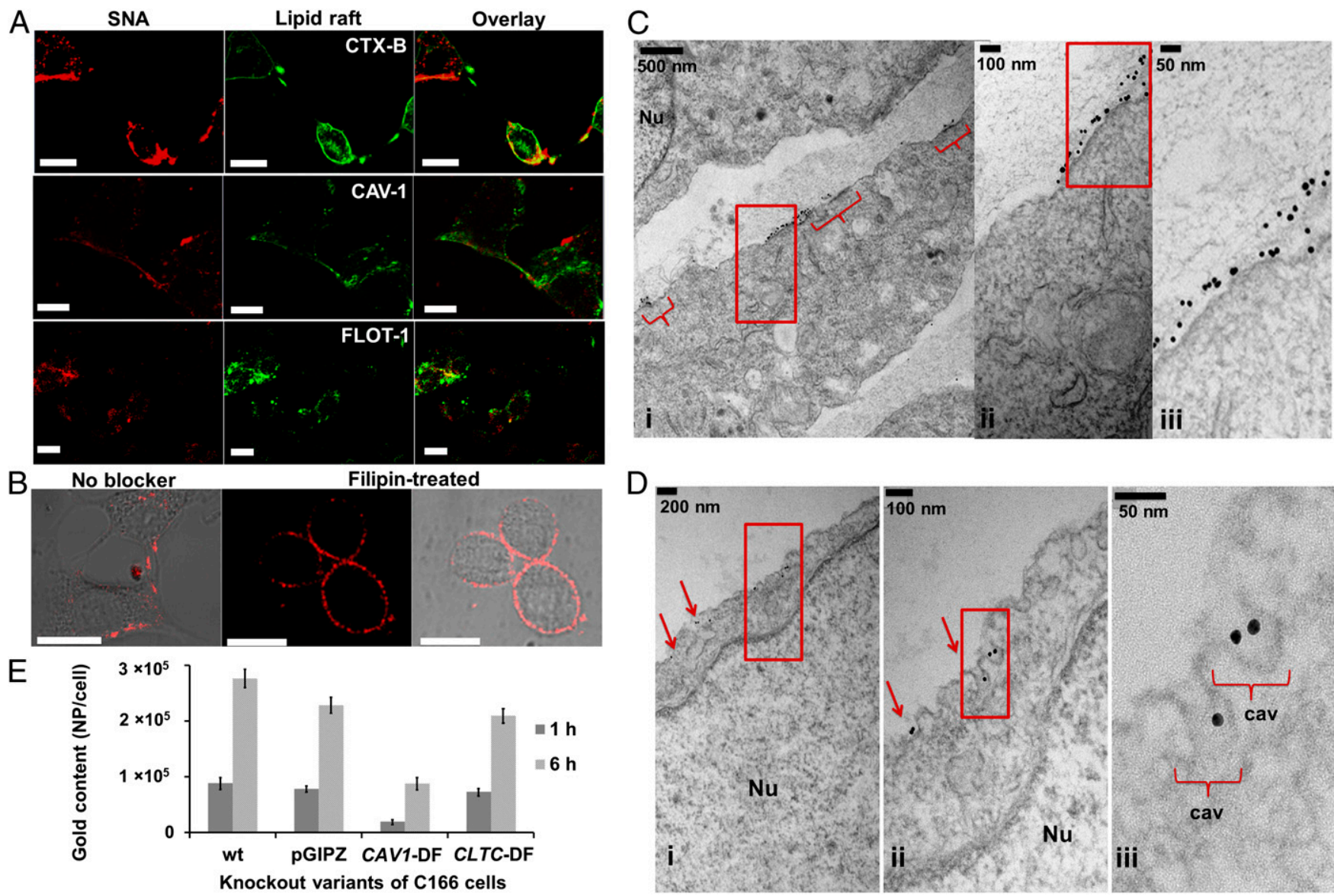

Fig. 3. Lipid-raft/caveolae-mediated endocytosis. (A) Confocal immunofluorescence shows colocalization of fluorescence signals from SNAs (red) and lipidraft resident proteins (green), including CTX-B, CAV-1, and FLOT-1, after $1 \mathrm{~h}$ of incubation of SNAs. The Manders overlap coefficient is $0.6-0.7$ for all proteins. $(\mathrm{Scale} b a r, 20 \mu \mathrm{m}$.) (B) SNAs never enter the cytosol of filipin-treated cells, as evidenced by the rings of fluorescence at the cell periphery. (Scale bar, $20 \mu \mathrm{m}$.) $(C)$ TEM images of cells incubated with SNAs for 5 min reveal lipid-raft microdomains. (i) The portion of the cell membrane (brackets) with higher SNA association exhibits darker staining intensity than other portions (ii; enlargement of the boxed area of $i$ ). There is positive correlation between the concentration of SNAs and membrane staining intensity. Intense staining may indicate the accumulation of transmembrane proteins at lipid-rafts (bracket). iii is the enlargement of the boxed area of ii. (D) TEM images of cells incubated with SNAs for 5 min provide evidence of caveolae-mediated endocytosis. (i) SNAs (arrows) collect along the cell membrane (ii; enlargement of the boxed area of $i$ ). Isolated SNAs are localized inside flask-shaped invaginations (iii; enlargement of the boxed area of ii). The size of each caveolae (cav)-like invagination is 50-100 nm in diameter. Nu, nucleus. (E) With CAV-1 silenced by an RNAi-mediated mechanism, CAV1-DF cells exhibit $60 \%$ reduction in the uptake of SNAs compared with that of pGIPZ-control cells. By contrast, CLTC-DF cells (with clathrin heavy chain silenced) only exhibit $\sim 10 \%$ reduction in uptake. Reported values represent mean \pm SE from the average of six independent experiments.

mediated endocytosis are CTX-B (which colocalizes with SNAs in lipid-rafts) and glycosylphosphatidylinositol (GPI)-anchored proteins (13). Indeed, pretreatment of cells with phosphatidylinositolphospholipase C, which enzymatically cleaves GPI-anchored proteins from the cell membrane (14), reduced the uptake of SNAs by $\sim 50 \%$ (Fig. S2B). By TEM imaging, after 5 min of incubation with C166 cells, individual SNAs are localized in flaskshaped invaginations of $50-100 \mathrm{~nm}$ in diameter at the cell membrane (Fig. 3D). To provide direct evidence of caveolae-mediated endocytosis, we generated stable $t$ cells with deficient expression levels of caveolin-1 (denoted "CAV1-DF") by lentiviral transfection of $\mathrm{C} 166$ cells with pGIPZ plasmids that contain shRNA sequences for silencing the expression of the $C A V 1$ gene (Figs. S3 and S4). Compared with pGIPZ-control cells, $C A V 1$-DF cells exhibited $\sim 60 \%$ reduction in endocytosis of SNAs (Fig. $3 E$ ). Thus, we conclude that lipid-rafts, or more specifically caveolae, significantly mediate the endocytosis of SNAs for C166 cells.

Endocytosis via Class A Scavenger Receptors. Literature precedent has indicated positive correlation between the degree of endocytosis of SNAs and loading density of oligonucleotides (15). Here, pretreatment of $\mathrm{C} 166$ cells with $\mathrm{NaN}_{3}$ reduced uptake of SNAs by $\sim 55 \%$, suggesting an energy-dependent nature of endocytosis (Fig. S5). Both observations led us to speculate the presence of cell surface receptors that support multivalent binding with the densely packed oligonucleotides of SNAs. We used ICP-MS to quantify the cellular association of SNAs after incubation for $15 \mathrm{~min}$, a duration of time long enough to allow for binding but not significant cellular uptake (Fig. 2D). Results from this assay resemble a typical Langmuir binding isotherm for ligand-receptor interactions (Fig. 4A). Next, we performed a competitive binding assay, in which C166 cells were incubated with a constant amount of SNA-AuNP conjugates $(10 \mathrm{nM})$ and varying amounts of ssDNAs or hollow SNAs for $15 \mathrm{~min}$. This assay allows one to probe the relative importance of multivalent binding involving SNAs; if hollow SNAs compete for surface receptors more effectively than ssDNAs, one can conclude that the polyvalent architecture of SNAs is critical for receptor binding. ICP-MS analysis of the gold content associated with the cells shows that the hollow SNAs are substantially more effective as inhibitors of SNA-AuNP conjugate binding to the cell surface under conditions where the total amount of oligonucleotide is held constant (Fig. 4B). Therefore, the 3D arrangement of the oligonucleotides contributes to its high binding affinity for cell surface receptors. 

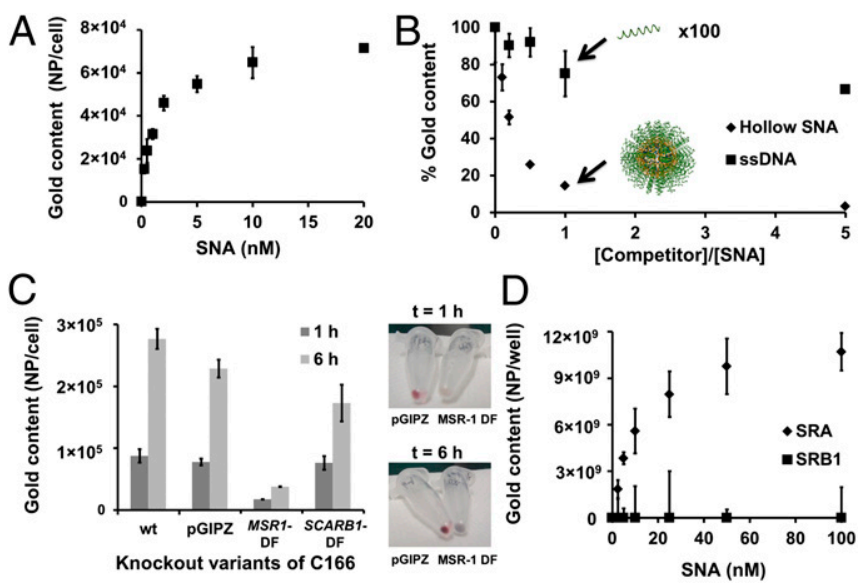

Fig. 4. Endocytosis via class A SRs. (A) The binding isotherm of SNAs to C166 cells resembles typical Langmuir kinetics, with a plateau indicative of the "receptor-limited" regime in the presence of excess SNAs. $(B)$ Incubation of cells with different molar ratios of hollow SNAs (with SNA concentration kept at $10 \mathrm{nM}$ ) for 15 min shows dose-dependent reduction in the cellular association of SNAs due to competition by hollow SNAs for cell surface receptors. ssDNAs are dramatically less effective as a competitor than hollow SNAs. (C) With their SR-A silenced by an RNAi-mediated mechanism, MSR1DF cells exhibit $80 \%$ reduction in the uptake of SNAs compared with that of pGIPZ-control cells. By contrast, SCARB1-DF cells (with their class B SR Type I silenced) only exhibit $\sim 25 \%$ reduction. Reported values represent mean $\pm \mathrm{SE}$ from the average of six independent experiments. $(D)$ From the modified ELISA, SNAs can bind to recombinant SR-A with a binding dissociation constant of $\sim 5 \mathrm{nM}$.

We previously reported a role for scavenger receptors (SRs) in mediating the endocytosis of SNAs using fucoidan (FCD) and polyinosinic acid (Poly I) as pharmacological inhibitors (16). Here, we verified that the pretreatment of C166 cells with FCD and Poly I resulted in $80-90 \%$ reduction in the uptake of SNAs (Fig. S5). As FCD and Poly I can bind to more than one member of the SR family $(17,18)$, they do not allow for the determination of the specific SR member(s) responsible for the endocytosis of SNAs. Based on this result, we can only speculate that class B SRs are not responsible, because FCD and Poly I are not ligands for this receptor subclass (18). To test this claim, we prepared stable C166 cells that have a deficient expression level of class B scavenger receptor type I, a representative member of the receptor subclass (denoted "SCARB1-DF") by lentiviral transfection of shRNAs targeting the $S C A R B 1$ gene. Compared with nontargeting pGIPZ-control cells, SCARB1-DF cells revealed only $25 \%$ reduction in uptake of SNAs (Fig. $4 C$ ). To explore the involvement of other SRs, we devised a modified ELISA to measure the binding affinity of SNAs to SRs (Fig. S6A). Class A SR (SR-A) demonstrates high affinity toward SNAs, netting an apparent binding dissociation constant of $\sim 5 \mathrm{nM}$ (Fig. $4 D$ ). By contrast, SR-BI does not exhibit appreciable binding to SNAs, in agreement with the uptake data from the use of pharmacological inhibitors (i.e., FCD and Poly I) and SCARB1-DF cells. These data led us to hypothesize the importance of SR-A in mediating the endocytosis of SNAs. We prepared C166 cells deficient in SR-A by lentiviral transfection of shRNAs targeting the macrophage scavenger receptor 1 ( $M S R 1$ ) gene (denoted "MSRl-DF") (Figs. S3 and S4). Upon incubation with SNAs, pellets collected from MSR1-DF cells are markedly less red than those from pGIPZ-control cells. Ensuing ICP-MS analysis of the same pellets revealed $\sim 80 \%$ decrease in uptake of SNAs by MSRl-DF cells in contrast to nontargeting pGIPZ-control cells (Fig. 4C). Since such a degree of decrease matches with that achievable via the use of FCD and Poly I, we conclude that SR-A is an important receptor subclass that mediates the endocytosis of SNAs. To confirm the ability of SR-A to recognize oligonucleotides anchored on SNAs as specific ligands, we incubated SNAs as well as AuNPs that are covalently attached with poly(ethylene glycol) chains (PEG-AuNPs) to different ELISA wells immobilized with recombinant SR-A in parallel. SNAs clearly demonstrate dosedependent binding to SR-As, whereas PEG-AuNPs do not (Fig. $\mathrm{S} 6 B)$. We also compared the ability of SNAs and ssDNAs of the same sequence to engage SR-A immobilized on the ELISA plate. Under conditions where the total amount of oligonucleotide is held constant, SNAs can bind to SR-A at least 10 times more strongly than ssDNAs (Fig. S6C), in agreement with the results from the competitive binding assay conducted using $\mathrm{C} 166$ cells (Fig. $4 B$ ). These data confirm that the $3 \mathrm{D}$ oligonucleotide shell, not the NP core, dictates the binding of SNAs to SR-A.

Collective Involvement of SR-A and Lipid-Raft Proteins. We further investigated the relationship between the pathway of endocytosis and the cell surface receptor subclass that collectively mediate the endocytosis of SNAs. To this end, we incubated C166 cells with biotin-labeled SNAs (biotin-SNAs) for $2 \mathrm{~h}$, and lysed them with a nondetergent-based method to preserve the native interactions between biotin-SNAs and any cellular proteins that became associated with the biotin-SNAs. To elute the associated proteins, we incubated streptavidin-coated microbeads with the cell lysate overnight, followed by gentle centrifugation of the microbead-lysate mixture to separate the pellet (which contains microbeads presumably attached to biotin-SNAs and their associated proteins) from the supernatant (Fig. $5 A$ ). Western blot analysis of the pellet and supernatant reveals enrichment of lipid-raft proteins (CAV-1 and FLOT-1) and SR-A in the pellet, but not the supernatant. To account for nonspecific binding of proteins onto the microbeads, we performed the identical precipitation assay using lysate from cells transfected with biotinfree SNAs (Fig. 5B). Western blot analysis of both lysate samples shows no obvious enrichment of lipid-raft proteins or SR-A in the pellet. These results suggest the collective involvement of
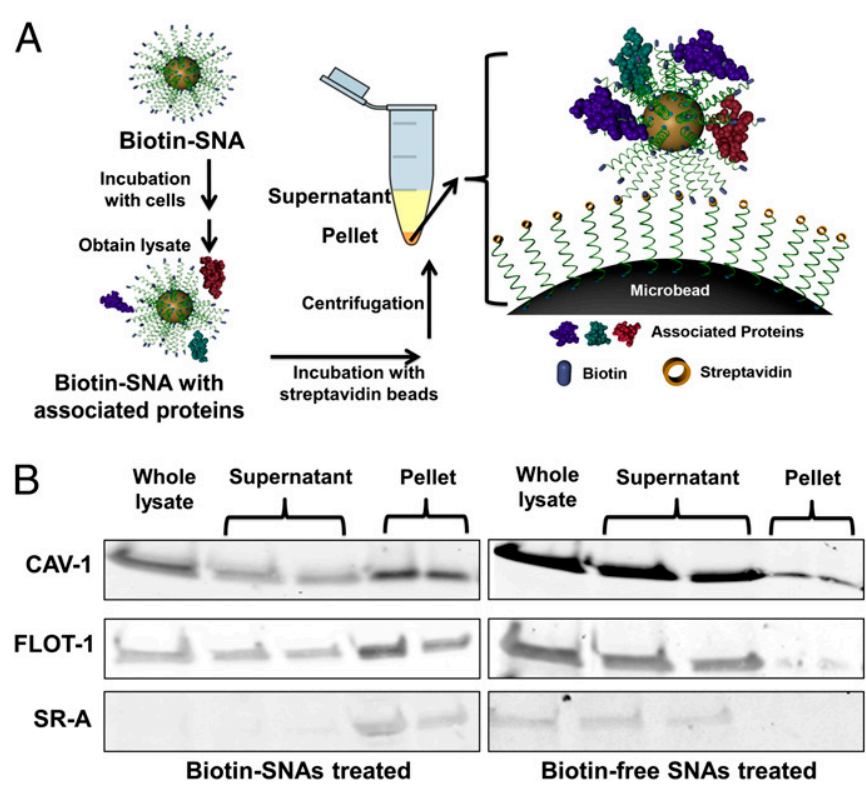

Fig. 5. Association between SR-A, lipid-raft proteins, and SNAs. (A) Schematic of the biotin-streptavidin protein precipitation assay. (B) Western blot analysis indicates enriched amounts of CAV-1, FLOT-1, and SR-A in the pellet but not the supernatant when $\mathrm{C} 166$ cells are incubated with biotin-labeled SNAs. By densitometric analysis, the gel bands for CAV-1, FLOT-1, and SR-A from the pellet fraction are 1.6-, 1.8-, and 7.5-fold more intense than those from the supernatant fraction, respectively. By contrast, for C166 cells that are incubated with the same concentration of biotin-free SNAs, enrichment of CAV-1, FLOT-1, and SR-A was only observed in the supernatant but not the pellet. Thus, SR-A and lipid-rafts collectively mediate the endocytosis of SNAs. 
lipid-rafts and SR-A in mediating the endocytosis of SNAs. In general, NPs or macromolecules can undergo various routes of endocytosis, depending upon their physicochemical parameters (e.g., size, surface charge, and composition), properties of their suspension medium (e.g., dosage concentration, serum proteins), as well as specific cell types tested $(19,20)$. In a similar vein as SNAs, several classes of extracellular entities [e.g., proteins (21), bacteria (22), and NPs (23)] can also enter cells via the lipid-raft/ caveolae pathway through the mediation of SRs.

Effect of CAV-1 and SR-A on Endocytosis of SNAs in Multiple Cell Types. The above studies strictly involve the use of C166 cells to illustrate the importance of CAV-1 and SR-A in mediating the endocytosis of SNAs. To establish the relevance of the same proteins for cell types other than $\mathrm{C} 166$, we measured the uptake kinetics of SNAs for other cell lines and compared the data to their expression of CAV-1 and SR-A. Besides C166, we selected A549 (human lung adenocarcinoma epithelium), 3T3 (mouse fibroblast), and HaCaT (human keratinocyte). These four cell lines possess a vast range of expression levels of CAV-1 and SRA, and indeed, pretreatment of these cells with FCD and Poly I led to pronounced reduction in their uptake of SNAs (Fig. $6 A$ ). Compared with A549 cells, HaCaT cells have a significantly higher expression level of both CAV-1 and SR-A. C166 and 3T3 cells exhibit intermediate levels of expression for the same proteins (Fig. 6B). By ICP-MS measurements, HaCaT cells exhibit the highest degree of endocytosis of SNAs. C166 and 3T3 cells show an intermediate degree of endocytosis, and A549 cells the lowest. Specifically, the uptake of SNAs in HaCaT cells is 4.8fold higher than that in A549 cells (Fig. 6C). These data indicate positive correlation between expression level of CAV-1 as well as SR-A and degree of endocytosis of SNAs, and underscore the importance of the same proteins in the endocytosis of SNAs in other cell types.

\section{Conclusions}

We have postulated a mechanism that explains the different cellular uptake properties between SNAs and their linear counterparts. For C166 cells, irrespective of the presence of
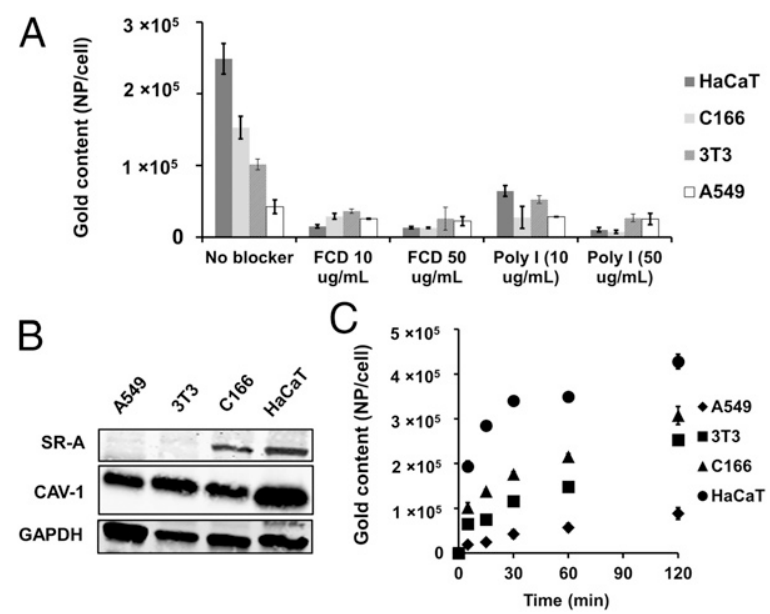

Fig. 6. Correlation between uptake of SNAs and expression level of CAV-1 and SR-A. (A) To probe the general importance of CAV-1 and SR-A, we selected three other cell lines besides C166 for additional investigation. They include HaCaT (immortal human keratinocyte), 3T3 (mouse fibroblast), and A549 (human lung epithelial adenocarcinoma). All are responsive to the treatment of FCD and Poly I, as evidenced by the drastic reduction in their uptake of SNAs. (B) Western blot was used to analyze the expression levels of CAV-1 and SR-A in four cell lines. In descending order of expression level, they are HaCaT, C166, 3T3, and A549. (C) These four cell lines exhibit different degrees of endocytosis of SNAs. HaCaT cells reveal the highest uptake, followed by C166, 3T3, and A549. the NP core, SNAs exhibit rapid cellular uptake kinetics and intracellular transport, whereas linear oligonucleotides do not. Moreover, SNAs can compete for cell surface receptors more substantially than linear oligonucleotides, due to the $3 \mathrm{D}$ arrangement of oligonucleotides. Specifically, SNAs can bind strongly to the SR-A, which, in turn, significantly mediate the cellular uptake of SNAs. Upon binding to cell surface SR-A, SNAs can enter cells via the caveolae-mediated pathway, presumably due to the close proximity of SNAs, SR-A, and lipid-raft microdomains. Since the expression level of SR-A and CAV-1 correlates positively with the degree of SNA uptake in cell types besides C166 (e.g., fibroblasts, epithelial cells, keratinocytes), we speculate that other cell types also operate under a similar mechanism of endocytosis as proposed for C166 (endothelial) cells. Results herein forge the foundation and reaffirm the potential for using SNA nanostructures as a universal platform for intracellular and in vivo applications, such as diagnostics and gene regulation, in different cell and tissue types.

\section{Materials and Methods}

Synthesis of Oligonucleotides. DNAs were synthesized on an MM48 Oligonucleotide synthesizer (BioAutomation) using standard solid-phase synthesis and reagents (Glen Research). All DNAs were purified using a ProStar high performance liquid chromatography (HPLC) instrument (Varian) with a Microsorb C18 column (Varian). Table S1 contains detailed sequence information of the DNAs.

Preparation of SNA Nanoconjugates. Thiolated DNAs were added to $10 \mathrm{~nm}$ citrate-capped AuNPs at a concentration of 1 OD of DNA per mL of $10 \mathrm{nM}$ AuNPs supplemented with $0.1 \%$ Tween 20 . After stirring at RT for $1 \mathrm{~h}$, the solution was aged with gradual additions of $\mathrm{NaCl}$ over $6 \mathrm{~h}$ to bring the final $\mathrm{NaCl}$ concentration to $0.5 \mathrm{M}$. Functionalized AuNPs were separated from free DNA strands via dialysis against Nanopure water using a 50-kDa Amicon molecular weight cutoff (MWCO) membrane (Millipore). AuNP and DNA concentrations were determined by measuring their extinction at $524 \mathrm{~nm}$ and $260 \mathrm{~nm}$, respectively, on a Cary 5000 ultraviolet/visible (UV-Vis) spectrophotometer (Agilent). Hollow SNAs were prepared based on published methods (3).

Generation of Stably Transfected Deficient Cells. Inoculated competent Escherichia coli strains with shRNA-expressing pGITZ vectors were purchased from the Lentiviral shRNA Libraries (Thermo Scientific) through the Northwestern Throughput/RNAi Core. The strains were incubated at $30{ }^{\circ} \mathrm{C}$ in ampicillin-containing LB broth for $24 \mathrm{~h}$ before extraction of plasmids using a QIAGEN Plasmid Plus Maxi Kit (catalog no. 12963). Lentiviral transduction was conducted in the DNA/RNA Delivery Core at Northwestern University. The transduced cells were selected and enriched in DMEM supplemented with $10 \%$ (vol/vol) FBS, $2 \mathrm{mM}$ L-glutamine, $100 \mathrm{U} / \mathrm{mL}$ penicillin, $100 \mu \mathrm{g} / \mathrm{mL}$ streptomycin, and $8 \mu \mathrm{g} / \mathrm{mL}$ puromycin.

Uptake Kinetics and Binding Assays. Seeded in a 24-well plate at a population of $5 \times 10^{4}$ cells per well $12 \mathrm{~h}$ in advance, cells were incubated with $0.3 \mathrm{~mL}$ of SNAs (10 nM in OptiMEM; Invitrogen) per well at $37{ }^{\circ} \mathrm{C}$ and $5 \% \mathrm{CO}_{2}$. To measure uptake kinetics, SNAs were removed at different time points, followed by OptiMEM rinses, trypsinization for counting using a hemacytometer, and centrifugation at $8,000 \mathrm{rpm}$ for $5 \mathrm{~min}$ to form a pellet for quantification by ICP-MS and imaging by TEM. For the direct binding assay, cells were incubated with different concentrations of SNAs for $15 \mathrm{~min}$. For the competitive binding assay, cells were incubated with a mixture that contains $10 \mathrm{nM}$ of SNAs and different concentrations of hollow SNAs for $15 \mathrm{~min}$. Cells were later pelleted for ICP-MS analysis.

ICP-MS. Cell pellets were digested with $0.3 \mathrm{~mL}$ of $3 \% \mathrm{HCl}$ in concentrated $\mathrm{HNO}_{3}$ at RT overnight. After adding $5 \mu \mathrm{L}$ of $5 \mathrm{ppm}$ indium (internal standard) and $5 \mathrm{~mL}$ of matrix solution $\left(2 \% \mathrm{HCl}\right.$ and $\left.2 \% \mathrm{HNO}_{3}\right)$, the Au-197 content of the resultant solution was measured by an X Series II ICP-MS (ThermoFisher) after subtracting the background gold content of untreated cells. Unless otherwise mentioned, reported values represent mean \pm SE from the average of three independent experiments.

TEM. Cell pellets were resuspended in $0.2 \mathrm{~mL}$ of molten $4 \%$ gelatin in PBS and pelleted again by centrifugation at 15,000 rpm for $5 \mathrm{~min}$. Embedded in congealed gelatin, cells were fixed in $2.5 \%$ glutaraldehyde in $100 \mathrm{mM}$ sodium cacodylate buffer $(\mathrm{pH}=7.4)$, stained by $1 \% \mathrm{OsO}_{4}$, and by $0.9 \%$ 
$\mathrm{OsO}_{4}$ and $0.3 \% \mathrm{~K}_{4} \mathrm{Fe}(\mathrm{CN})_{6}$, with all steps carried out at $4{ }^{\circ} \mathrm{C}$ for $2 \mathrm{~h}$. After gradual dehydration with ethanol and propylene oxide, cell pellets were embedded in Epon 812 resins (Electron Microscopy Sciences). We deposited 80-nm-thick sections on 200-mesh copper grids (Electron Microscopy Sciences) and stained with $3 \%$ uranyl acetate (SPI Supplies) and Reynolds lead citrate for visualization under a JEM 1230 microscope (JEOL) using a beam voltage of $80 \mathrm{kV}$. An Orius SC 1000 CCD camera (Gatan) was used to record the images.

Confocal Microscopy and Immunofluorescence. Seeded in a $35 \mathrm{~mm}$ FluoroDish (World Precision Instruments), cells were incubated with $10 \mathrm{nM}$ of Cy5-SNAs in OptiMEM for different time points. Cells were rinsed with PBS, fixed in $3.7 \%$ paraformaldehyde (PFA) in PBS for $15 \mathrm{~min}$, and imaged under a Zeiss LSM 510 inverted confocal scanning microscope. The excitation wavelength of Cy5-SNAs was $633 \mathrm{~nm}$, and the corresponding emission filter was 660-710 $\mathrm{nm}$. To track the colocalization of SNAs with cellular proteins, after incubation with $10 \mathrm{nM}$ Cy5-SNAs for $1 \mathrm{~h}$, cells were rinsed with PBS, fixed in $3.7 \%$ PFA in PBS, and permeated with $0.1 \%$ Triton- 100 for 10 min. After blocking with $2 \%$ BSA in PBS for $1 \mathrm{~h}$, cells were stained with a primary antibody against the protein marker of interest at $5 \mu \mathrm{g} / \mathrm{mL}$ ( $1 \%$ BSA in PBS) overnight at $4{ }^{\circ} \mathrm{C}$. If necessary, after rinses with $0.05 \%$ Tween- 20 in PBS, cells were stained with an AlexaFluor 488-labeled secondary antibody [Invitrogen, Alexa Fluor 488 Goat Anti-Rabbit lgG (H+L)] at $1 \mu \mathrm{g} / \mathrm{mL}$ (1\% BSA in PBS) for $1 \mathrm{~h}$ at RT. The excitation wavelength of the secondary antibody was $488 \mathrm{~nm}$, and the corresponding emission filter was $500-550 \mathrm{~nm}$. The primary antibodies include rabbit against EEA1 (Abcam ab2900), rabbit against CAV-1 (Cell signaling 3238), and rabbit against FLOT-1 (Santa Cruz Biotechnology sc-25506). To label lipid-raft microdomains, cells were first incubated with Cy5-SNAs for $1 \mathrm{~h}$, rinsed gently with OptiMEM, and further incubated with AlexaFluor 488-labled CTX-B (Molecular Probes C-34775; $8 \mu \mathrm{g} / \mathrm{mL}$ in OptiMEM) for $20 \mathrm{~min}$ at $37^{\circ} \mathrm{C}$, followed by fixation in $3.7 \%$ PFA for $15 \mathrm{~min}$. To measure the extent of colocalization between the fluorescence signals of SNAs and protein markers, the Zen Digital Imaging (Zeiss) software allows for the calculation of the Manders overlap coefficient (24). An overlap coefficient of higher than 0.6 indicates colocalization (25).

Chemical Blocking Studies. In 24-well plates, cells were pretreated with $280 \mu \mathrm{L}$ of OptiMEM that contains different concentrations of chemical blockers per well for $30 \mathrm{~min}$. We added $20 \mu \mathrm{L}$ of $150 \mathrm{nM}$ SNAs into each well, and cells were incubated for another $30 \mathrm{~min}$. To measure the extent of blocking, cells were pelleted and digested for ICP-MS analysis. To visualize the extent of blocking, cells in eight-well Lab-Tek II chamber coverglass system were pretreated by $280 \mu \mathrm{L}$ of chemical blocker solution. We added $20 \mu \mathrm{L}$ of $150 \mathrm{nM}$ Cy5-SNAs for confocal imaging. Table S2 presents the concentration and pharmacological action of the chemical blockers.

1. Rosi NL, et al. (2006) Oligonucleotide-modified gold nanoparticles for intracellular gene regulation. Science 312(5776):1027-1030.

2. Cutler Jl, Auyeung E, Mirkin CA (2012) Spherical nucleic acids. J Am Chem Soc 134(3): 1376-1391.

3. Cutler Jl, et al. (2011) Polyvalent nucleic acid nanostructures. J Am Chem Soc 133(24): 9254-9257.

4. Seferos DS, Giljohann DA, Hill HD, Prigodich AE, Mirkin CA (2007) Nano-flares: Probes for transfection and mRNA detection in living cells. J Am Chem Soc 129(50): 15477-15479.

5. Giljohann DA, Seferos DS, Prigodich AE, Patel PC, Mirkin CA (2009) Gene regulation with polyvalent siRNA-nanoparticle conjugates. J Am Chem Soc 131(6):2072-2073.

6. Mayor S, Pagano RE (2007) Pathways of clathrin-independent endocytosis. Nat Rev Mol Cell Biol 8(8):603-612.

7. Wang LH, Rothberg KG, Anderson RG (1993) Mis-assembly of clathrin lattices on endosomes reveals a regulatory switch for coated pit formation. J Cell Biol 123(5): 1107-1117.

8. Le Roy C, Wrana JL (2005) Clathrin- and non-clathrin-mediated endocytic regulation of cell signalling. Nat Rev Mol Cell Biol 6(2):112-126.

9. Allen JA, Halverson-Tamboli RA, Rasenick MM (2007) Lipid raft microdomains and neurotransmitter signalling. Nat Rev Neurosci 8(2):128-140.

10. Barnakov AN (1994) Sequential treatment by phosphotungstic acid and uranyl acetate enhances the adherence of lipid membranes and membrane proteins to hydrophobic EM grids. J Microsc 175(Pt 2):171-174.

11. Rothberg KG, et al. (1992) Caveolin, a protein component of caveolae membrane coats. Cell 68(4):673-682.

12. Parton RG, Simons K (2007) The multiple faces of caveolae. Nat Rev Mol Cell Biol 8(3): 185-194.

13. Doherty GJ, McMahon HT (2009) Mechanisms of endocytosis. Annu Rev Biochem 78(1):857-902
Western Blotting. Cells were grown to $10^{6}$ cells per T-75 flask (Corning) before collection. Cells were trypsinized, and pellets were resuspended in $500 \mu \mathrm{L}$ of $1 \times$ mammalian cell lysis buffer (Cell Signaling) containing $1 \times$ protease and phosphatase inhibitor (Thermo Scientific). The whole-cell lysates were then clarified by centrifugation for $5 \mathrm{~min}$ at $15,000 \mathrm{rpm}$ at $4{ }^{\circ} \mathrm{C}$. Protein concentrations were determined using the BCA Protein Assay Kit (Pierce). Equal amounts $(40 \mu \mathrm{g})$ of protein samples were fractionated by a $4-20 \%$ precast gradient gel (Bio-Rad), transferred to nitrocellulose membranes (Thermo Scientific), and blocked in Odyssey blocking buffer (LI-COR Biosciences). SR-A was probed by primary rat antibody (1:500) (Pierce Antibodies MA1-81060) and CAV-1 was probed by primary rabbit antibody (1:500) (Cell Signaling $3267)$, together with primary rabbit antibody against GAPDH $(1: 1,000)$ (Santa Cruz Biotechnology sc-25778). The desired bands were detected by labeling with anti-rat $(1: 5,000)$ or anti-rabbit $(1: 10,000)$ IgG-IRDye $680 \mathrm{sec}$ ondary antibodies and visualized using the Odyssey CLx Infrared Imaging System (LI-COR Biosciences).

Protein Precipitation Assay. After incubation with biotin-SNAs for $2 \mathrm{~h}$, cells were rinsed with PBS and homogenized in ice-chilled, $0.5 \mathrm{M} \mathrm{Na}_{2} \mathrm{CO}_{3}$ containing $1 \times$ protease and phosphatase inhibitor (Thermo Scientific). The homogenate was transferred to a microcentrifuge tube and incubated with the same volume of prewashed streptavidin-coupled Dynabeads (Invitrogen) at $4{ }^{\circ} \mathrm{C}$ overnight. Beads and associated proteins were collected by centrifugation at 5,000 rpm for $1 \mathrm{~min}$ and resuspended in DTT-containing loading buffer. After boiling for $5 \mathrm{~min}$, samples were fractionated by a 4-20\% precast gradient gel (Bio-Rad), followed by Western blotting of SR-A, CAV-1, and FLOT-1.

ACKNOWLEDGMENTS. We thank Charlene Wilke of Biological Imaging Facility (BIF) for assistance in ultramicrotomy and Alex Yemelyanov (DNA RNA Delivery Core, Skin Disease Research Center, Northwestern University) for preparing lentivirally transduced deficient cells. ICP-MS measurements were performed at the Quantitative Bioelemental Imaging Center (Northwestern University) and confocal and TEM imaging were both performed at the BIF (Northwestern University). C.A.M. acknowledges support from the Centers of Cancer Nanotechnology Excellence (CCNE) initiative of National Institutes of Health (NIH) Awards U54 CA119341 and U54 CA151880, Dixon Translational Research Grants Initiative, the Nanoscale Science and Engineer ing Centers (NSEC) initiative of National Science Foundation Award EEC 0647560, the Prostate Cancer Foundation, NIAMS/NIH Grants R01AR060810 and R21AR062898, and Defense Advanced Research Planning Agency/Microsystems Technology Office (MTO) Award N66001-11-1-4189. C.H.J.C. acknowledges a postdoctoral research fellowship from The Croucher Foundation. L.H. is a Howard Hughes Medical Institute International Student Research Fellow and acknowledges a Ryan Fellowship from Northwestern University. Both S.P.N. and E.A. acknowledge a National Defense Science and Engineering Graduate (NDSEG) Fellowship.

14. Sharom FJ, Lehto MT (2002) Glycosylphosphatidylinositol-anchored proteins: Structure, function, and cleavage by phosphatidylinositol-specific phospholipase C. Biochem Cell Biol 80(5):535-549.

15. Giljohann DA, et al. (2007) Oligonucleotide loading determines cellular uptake of DNA-modified gold nanoparticles. Nano Lett 7(12):3818-3821.

16. Patel PC, et al. (2010) Scavenger receptors mediate cellular uptake of polyvalent oligonucleotide-functionalized gold nanoparticles. Bioconjug Chem 21(12):2250-2256.

17. Palecanda A, et al. (1999) Role of the scavenger receptor MARCO in alveolar macrophage binding of unopsonized environmental particles. J Exp Med 189(9): 1497-1506.

18. Platt N, Gordon S (1998) Scavenger receptors: Diverse activities and promiscuous binding of polyanionic ligands. Chem Biol 5(8):R193-R203.

19. Dobrovolskaia MA, McNeil SE (2007) Immunological properties of engineered nanomaterials. Nat Nanotechnol 2(8):469-478.

20. Nel AE, et al. (2009) Understanding biophysicochemical interactions at the nano-bio interface. Nat Mater 8(7):543-557.

21. Schnitzer JE, Oh P, Pinney E, Allard J (1994) Filipin-sensitive caveolae-mediated transport in endothelium: Reduced transcytosis, scavenger endocytosis, and capillary permeability of select macromolecules. J Cell Biol 127(5):1217-1232.

22. Zhu X-D, et al. (2011) Caveolae-dependent endocytosis is required for class $A$ macrophage scavenger receptor-mediated apoptosis in macrophages. $J \mathrm{Biol}$ Chem 286(10):8231-8239.

23. Zhang LW, Monteiro-Riviere NA (2009) Mechanisms of quantum dot nanoparticle cellular uptake. Toxicol Sci 110(1):138-155.

24. Manders EMM, Verbeek FJ, Aten JA (1993) Measurement of co-localization of objects in dual-colour confocal images. J Microsc 169(3):375-382.

25. Zinchuk V, Zinchuk $O$ (2001) Quantitative colocalization analysis of confocal fluorescence microscopy images. Curr Protoc Cell Biol 39:4.19.1-4.19.16. 